\title{
Exocrine System
}

National Cancer Institute

\section{Source}

National Cancer Institute. Exocrine System. NCI Thesaurus. Code C12957.

A system of glands that secrete fluids delivered via ducts and tubes directly to org ans

including the skin. Examples include the exocrine pancreas and the prostate gland. 